\title{
CT perfusion for response evaluation after interventional ablation of hepatocellular carcinoma: a prospective study
}

\author{
Mohamed Fouad Osman ${ }^{1}$, Islam H. Shawali ${ }^{*}$ (D), Lamiaa I. A. Metwally ${ }^{1}$, Ahmed Hosni Kamel ${ }^{1}$ and \\ Mohamed El Sherbiny Ibrahim²
}

\begin{abstract}
Background: Computed tomography (CT) perfusion was found to be useful in assessing treatment response in a variety of cancers through the evaluation in the arterial perfusion changes. We investigated the performance of CT perfusion parameters for assessment of hepatocellular carcinoma (HCC) response to radiofrequency ablation (RFA) and trans-arterial chemoembolization (TACE). We conducted a prospective diagnostic test accuracy study that recruited 70 HCC patients who were scheduled to undergo TACE or RFA. For each dynamic CT scan acquisition, four single perfusion CT image maps were generated, including functional maps of blood flow (BF), blood volume (BV), mean transit time (MTT), and permeability surface (PS).
\end{abstract}

Results: In TACE-treated lesions, the BV achieved a sensitivity and specific of $100 \%$ and $83.3 \%$, at a cutoff level of $\leq 122 \mathrm{ml} / \mathrm{min} / 100 \mathrm{gm}$, for responders. Likewise, at a cutoff level of $>10 \mathrm{~s}$, transit time had a sensitivity of $90.5 \%$ and specificity of $100 \%$. At a cutoff level of $\leq 14 \mathrm{ml} / \mathrm{min} / 100 \mathrm{gm}$, the PS had a sensitivity of $100 \%$ and specificity of $83.33 \%$ for responders. In RFA-treated lesions, at a cutoff level of $\leq 170 \mathrm{ml} / \mathrm{min} / 100 \mathrm{gm}$ and $\leq 11 \mathrm{ml} / 100 \mathrm{gm}$, the BF and BV had a sensitivity of $100 \%$ and specificity 100\%, respectively, for responders. At a cutoff level of $\leq 11 \mathrm{ml} / \mathrm{min} / 100 \mathrm{gm}$, PS had a sensitivity $77.27 \%$ and specificity $80 \%$.

Conclusions: The present study confirms the feasibility of CT perfusion for assessment of response to TACE and RFA among patients with HCC.

Keywords: CT perfusion, Hepatocellular carcinoma, Intervention ablation, Response

\section{Background}

Hepatocellular carcinoma (HCC) is the sixth most common malignancy and a significant cause of mortality worldwide. According to recent epidemiological figures, the incidence of $\mathrm{HCC}$ shows a steady increase, with more than 900,000 new cases of HCC diagnosed in 2020; besides, nearly 830,000 global deaths were attributed to HCC in 2020 [1]. East Asia harbors the largest number of $\mathrm{HCC}$ cases across the globe, while the lowest incidence is

\footnotetext{
*Correspondence: Islamshawali@gmail.com; eslam.a.samy@kasralainy.edu.eg 1 Diagnostic and Interventional Radiology, Cairo University, Cairo, Egypt Full list of author information is available at the end of the article
}

reported in South-central Asia [2]. In Egypt, HCC represents a public health and financial burden and accounted for nearly 25,400 cases in 2018, ranking it as the most prevalent cancer in the Eastern Mediterranean country $[3,4]$. HCC is the most common form of hepatic malignancy, and it affects mainly males older than 50 years old [5]. The development of $\mathrm{HCC}$ is attributed to a wide range of risk factors, including chronic hepatitis $B$ and $C$, non-alcoholic steatohepatitis, and autoimmune hepatitis, as well as genetic predisposition [6]. While the underlying pathogenesis of HCC has not been fully elucidated yet, the cumulative body of evidence has improved our understanding of the molecular pathways, such as 
CCNA2 or CCNE1, involved in the invasive development of HCC [7]. Patients with HCC are prone to a wide range of complications, whose severity increases proportionally with advanced disease stages, such as liver failure, variceal bleeding, treatment-related complications, and death $[8,9]$. In Egypt, HCC is the most common cause of cancer-related deaths ( $25 \%)$, which calls for effective surveillance and preventive strategies [4].

Currently, the management of HCC relies mainly in disease stages, with ablation and trans-arterial chemoembolization offered to patients with early and intermediate stages [10]. According to several clinical trials, RFA and TACE had significantly improved the outcomes of survival of HCC patients, with manageable safety profile [11]. Thus, both techniques are the most common approaches for HCC management in many centers. Nonetheless, assessment of patients' response to RFA/ TACE is essential step in the management algorithms of HCC [2]. While the Response Evaluation Criteria in Solid Tumors (RECIST) is the most commonly utilized evaluation form in many malignancies, previous reports showed that the RECIST has many limitations in the setting of HCC [12]. Therefore, several imaging-based modalities were proposed for accurate evaluation of patients' response to loco regional treatment.

Recently, computed tomography (CT) perfusion has emerged as a useful imaging modality for quantitative evaluation of tumor angiogenesis [13]. In the setting of $\mathrm{HCC}$, it was found that the changes in the CT perfusion dynamic parameters correlated significantly with the hepatic pathological changes [14]. Thus, CT perfusion demonstrated fair diagnostic accuracy for diagnosis of HCC [15]. Moreover, CT perfusion was found to be useful in assessing treatment response in variety of cancers through the evaluation in the arterial perfusion changes. However, limited data are available regarding the performance of CT perfusion parameters for evaluation of HCC response to RFA and TACE. Thus, we investigated the performance of CT perfusion parameters for assessment of HCC response to RFA and TACE.

\section{Methods}

The present manuscript was approved by the local ethics committee of Cairo University Hospital [D-23-2019] and prepared in concordance with the recommendations of STrengthening the Reporting of OBservational studies in Epidemiology (STROBE) [16].

\section{Study design and patients}

We conducted a prospective diagnostic test accuracy study that recruited 70 HCC patients who were scheduled to undergo TACE or RFA through the period from January 2019 to December 2020 at the Cairo university hospitals. Adult patients (older than 18 years) were included, with no gender restriction, if they had an established diagnosis of focal or multifocal HCC diagnosed by histopathology or CT findings. Only patients with ChildPugh class A or B disease were included. We excluded patients with relative or absolute contraindications to CT imaging or intravenous (IV) contrast. Patients were required to sign written informed consent before the study's initiation.

\section{Data collection and CT perfusion}

Eligible patients underwent detailed history and data collection regarding the age, sex, clinical status, stage of liver cirrhosis according to Child-Pugh, etiology of HCC, date of intervention, type of intervention, size, number and location of lesions, laboratory parameters (blood screen, bilirubin, transaminase, urea, creatinine, alfa feto protein level), extrahepatic spread of the disease.

In patients who underwent TACE, we collected selectiveness of TACE, the use of microcatheter, type and size of embolization particles, the dose of chemotherapeutic agent, number of TACE procedures, response to treatment according to mRECIST criteria and survival.

All patients underwent multi-detector CT (MDCT) and $\mathrm{CT}$ perfusion examination $3 \pm 1.3$ months post-intervention.

For MDCT, we utilized a 16-slice MDCT scanner (Alexion, Toshiba Medical Systems, Japan), alongside with injection of $100-120 \mathrm{~mL}$ of non-ionic iodinated CM (Ultravist ${ }^{\circledR}$ (brand of iopromide) injection 300; Bayer, Berlin, Germany). The scan passed through an unenhanced phase, followed by a post-contrast triphasic acquisition.

CTP was performed using the same scanner, after the multiphase MDCT study. Before injection of CM, we selected the appropriate transverse level in order to include the maximal diameter of the treated HCC lesion, according to the unenhanced scan of the prior CECT study. Care was taken to ensure that $2 \mathrm{~cm}$ of the target lesion was included for the cine acquisition. Both the tumor location and anatomic landmarks (such as portal vein and aorta) were used in the decision. In patients with multiple lesions who underwent TACE, we chose the largest lesion and considered it as the target lesion.

The dynamic image acquisition includes first initial phase study and delayed phase study. The first phase study is composed of images acquired during the initial phase of contrast agent administration within 40-60 s. The second delayed phase study is $2-10 \mathrm{~min}$ after the first phase study. The following CT parameters were used to acquire perfusion data: image acquisition for a total duration of 40-60 s with one image every $1 \mathrm{~s}$ starting $1 \mathrm{~s}$ after injection of $40-50 \mathrm{ml}$ of contrast at a rate 
of 4-7 ml/s with a tube current of $50-100 \mathrm{mAs}$, delayed phase involves acquisition of $1 \mathrm{~s}$ images acquired every $10 \mathrm{~s}$ for $2 \mathrm{~min}$. Every patient was clearly instructed to breathe normally during images acquisition and to avoid a deep breath when experiencing a hot flush sensation, commonly associated with the rapid bolus of iodinated $\mathrm{CM}$. Moreover, to avoid motion artifacts and images mis-registration,

Data were processed at a workstation (Advantage Windows 4.0; GE Medical Systems) with CTP software (GE Perfusion 3.0) by 2 radiologists. The parametric map images were created using the highest spatial resolution pixel-by-pixel calculation technique. Tissue perfusion valuation was based on the maximum slope model and using deconvolutional analysis as the average slope of the tissue enhancement divided by the peak enhancement in the aorta. ROIs were drawn, respectively, within surrounding cirrhotic liver parenchyma, trying to avoid arterial and portal vessels. The resulting temporal changes in contrast enhancement were then analyzed to quantify a range of parameters that reflected the functional status of tissue perfusion. For each dynamic CT scan acquisition, 4 single perfusion CT image maps were generated, including functional maps of blood flow (BF), blood volume (BV), mean transit time (MTT), and permeability surface (PS). To draw region of interest (ROI), the readers manually drew ROI on each CTP map and corresponding CTP parameters (BF, BV, MTT, and PS), values were averaged for all ROIs in each patient. ROI included at least two-thirds of the area of the HCC. Functional maps were represented in a color-coding scheme in rainbow format ranging from blue to red so as to obtain comparable color maps as varying shades of blue, green, yellow, and red in order of increasing perfusion.

Two hepatic imaging experienced readers evaluated CT perfusion data. The readers independently reviewed hepatic focal lesion perfusion parameters. Acquisition date and participant identification were removed from all images. The radiologists were blinded to all clinical information.

\section{Study's outcomes}

The primary outcome in the present study was the diagnostic accuracy of CT perfusion parameters in assessment of responders to TACE and RFA. The secondary outcomes included comparing CT perfusion parameters of TACE/RFA responders and recurrent activity between ablated lesions and cirrhotic parenchyma.

\section{Statistical analysis}

SPSS version 19 (SPSS Inc., Cary, NC) was used for the statistical analysis. We described the central tendency and variation in age and $\mathrm{CT}$ perfusion parameters using mean and standard deviation (SD). Frequency tables were used for gender and response to TACE/RFA. The association between response to TACE/RFA and CT perfusion parameters was assessed using an Independent $t$ test. Spearman correlation test was used to test the correlation between CT perfusion parameters and age. The diagnostic performance of $\mathrm{CT}$ perfusion parameters to predict TACE/RFA responders was evaluated using Receiver Operating Characteristic (ROC) curve analysis. A P-value of less than $5 \%$ was considered statistically significant.

\section{Results}

A total of 70 patients were included with a mean age of $66.83 \pm 5.99$ years old and male predominance $(91.4 \%)$. The underlying cause of cirrhosis in the patients was hepatitis $\mathrm{C}$-virus. The size of target lesions under assessment post-therapy was $3.5 \pm 1.4 \mathrm{~cm}, 54$ of which were located in the right hepatic lobe different segments; 64 patients were investigated after their first locoregional treatment session while the rest had several sessions before $3 \pm 1$.

Of the total patients, 33 patients (47.1\%) underwent TACE, and the rest of the patients underwent RFA. Among the 33 patients who underwent TACE, 31 patients underwent conventional TACE while the other 2 underwent TACE with drug eluding beads.

Treatment response of the target lesions was described according to CT perfusion results as responder and recurrent lesion activity based on perfusion parameters, and those results were confirmed through correlation with four phase CT or MRI studies based on mRECIST criteria as well as correlation with tumor markers levels.

Out of the patients who underwent TACE, 21 patients (64\%) were classified as responders, compared to $59.5 \%$ of the patients who underwent RFA (Fig. 1A, B).

Among patients who underwent TACE, there was statistically significant lower BF in responders versus recurrent cases $(46.05 \pm 39.71$ vs. $292.79 \pm 150.16 \mathrm{ml} /$ $\mathrm{min} / 100 \mathrm{gm} ; p<0.001$ ) (Figs. 2, 3, 4). Likewise, there was statistically significant higher mean transit time in responders versus recurrent cases $(20.65 \pm 10.8$ vs. $6 \pm 2.78 \mathrm{~s} ; p<0.001)$. Responders had significantly lower permeability $(5.58 \pm 5.67$ vs. $23.05 \pm 9.5 ; p<0.001)$ and higher $\mathrm{HU}$ on non-contrast, arterial, and porto-venous $(p<0.05)$. Among patients who underwent RFA, there was statistically significant lower BF $(61.7 \pm 54.5$ vs. $565.8 \pm 257.3 \mathrm{ml} / \mathrm{min} / 100 \mathrm{gm} ; p<0.001)$ and lower BV $(5.5 \pm 2.9$ vs. $47.5 \pm 10.03 \mathrm{ml} / 100 \mathrm{gm} ; p<0.001)$. in responders versus recurrent cases. Likewise, there was a statistically significant higher arterial fraction in responders versus recurrent cases $(0.54 \pm 0.23$ vs. $0.34 \pm 0.3 ; p=0.031)$. Responders had significantly lower 


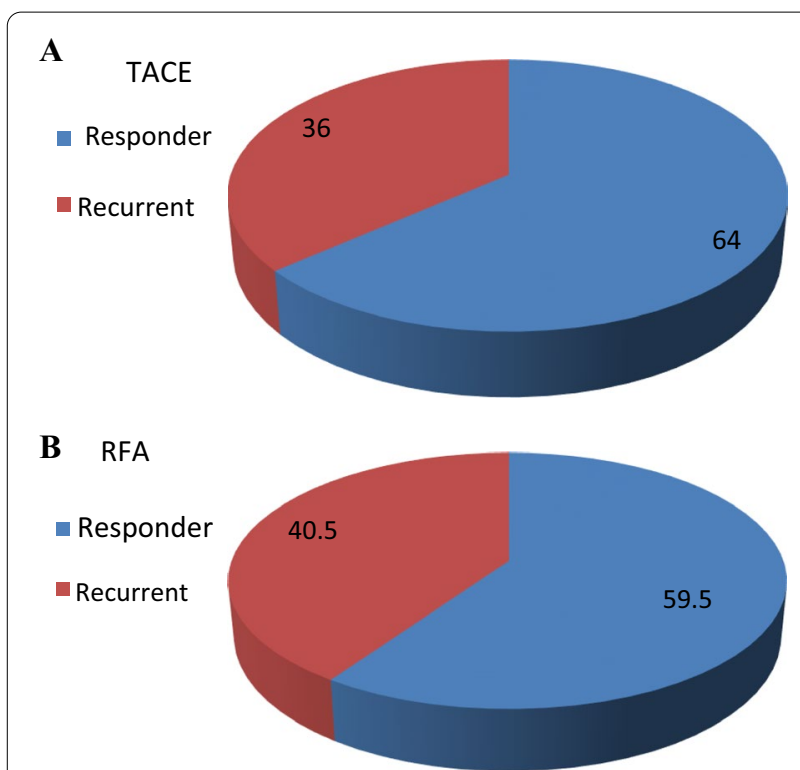

Fig. 1 Frequency of responders to TACE $(\mathbf{A})$ and RFA (B)

permeability $(10.3 \pm 9.1 \mathrm{vs} / 24.8 \pm 11.9 ; p<0.001)$ and higher $\mathrm{HU}$ on arterial and porto-venous $(p<0.05)$, as shown in Table 1.
Among responders to TACE, there were statistically significant lower BF $(p<0.001)$, BV $(p 0.001)$, mean transit time $(p<0.001)$, PS $(p<0.001)$, and higher arterial fraction $(p<0.001)$ and HU on non-contrast, arterial, and porto-venous $(p<0.05)$. Among responders to RFA, there were statistically significant lower BF $(61.7 \pm 54.5$ versus $565.8 \pm 257.3 \mathrm{ml} / \mathrm{min} / 100 \mathrm{gm} ; p<0.001)$ and lower $\mathrm{BF}$ $(p<0.001), \mathrm{BV}(p 0.001)$, PS $(p<0.001)$, and higher HU on non-contrast, arterial, and porto-venous $(p<0.05)$, as shown in Table 2.

In TACE-treated lesions, the BV achieved a sensitivity and specificity of $100 \%$ and $83.3 \%$, at a cutoff level of $\leq 122 \mathrm{ml} / \mathrm{min} / 100 \mathrm{gm}$, for responders. Likewise, at a cut-off level of $>10 \mathrm{~s}$, transit time had a sensitivity of $90.5 \%$ and specificity of $100 \%$. At a cut-off level of $\leq 14 \mathrm{ml} / \mathrm{min} / 100 \mathrm{gm}$, the PS had a sensitivity of $100 \%$ and specificity of $83.33 \%$ for responders. The HU noncontrast and arterial also had high sensitivity $(100 \%$ and $90.8 \%$, respectively) and specificity (100\%) for responders. Lastly, at a cut-off level of $>98$, HU porto-venous had a sensitivity of $95.24 \%$ and specificity of $100 \%$ (Table 3 ).

In RFA-treated lesions, at a cut-off level of $\leq 170 \mathrm{ml} /$ $\mathrm{min} / 100 \mathrm{gm}$ and $\leq 11 \mathrm{ml} / 100 \mathrm{gm}$, the BF and BV had a sensitivity of $100 \%$ and specificity $100 \%$, respectively, for responders. At a cut-off level of $\leq 11 \mathrm{ml} / \mathrm{min} / 100 \mathrm{gm}$, PS had a sensitivity of $77.27 \%$ and specificity of $80 \%$.
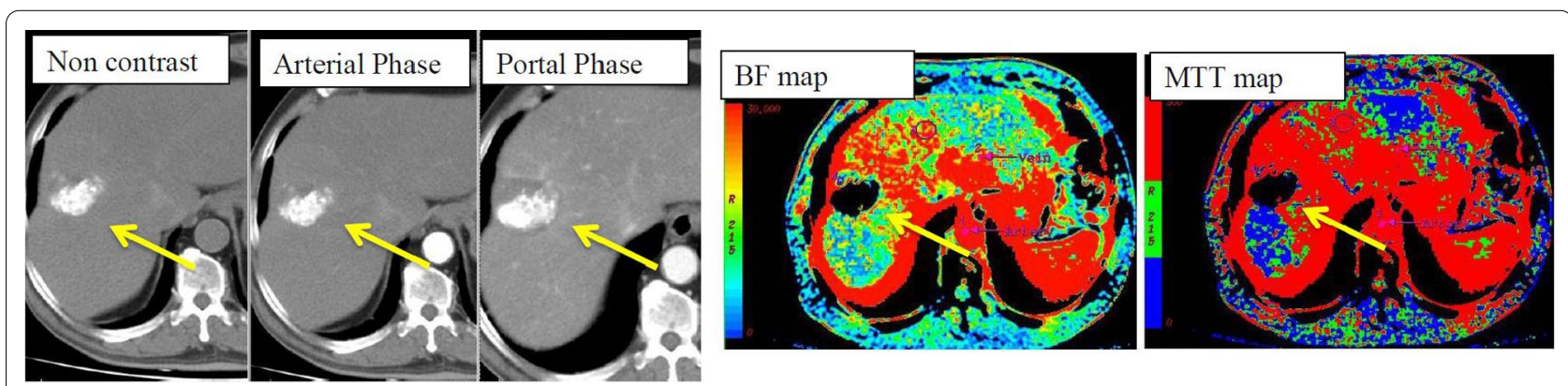

Fig. 2 69-year-old male patient with liver cirrhosis and right hepatic lobe segment VIII HCC underwent chemoembolization on June 2019. Follow-up post-intervention triphasic CT was done on September 2019 and revealed dense injected chemotherapy material "lipidol" showing no evidence of pathological enhancement. CTP functional maps showed intra lesional BF of $102 \mathrm{~mL} / 100 \mathrm{~g} / \mathrm{min}$ compared to $187 \mathrm{~mL} / 100 \mathrm{~g} / \mathrm{min}$ in the hepatic parenchyma. In addition, the mean transit time is almost doubled at the lesion measuring about $6 \mathrm{~s}$
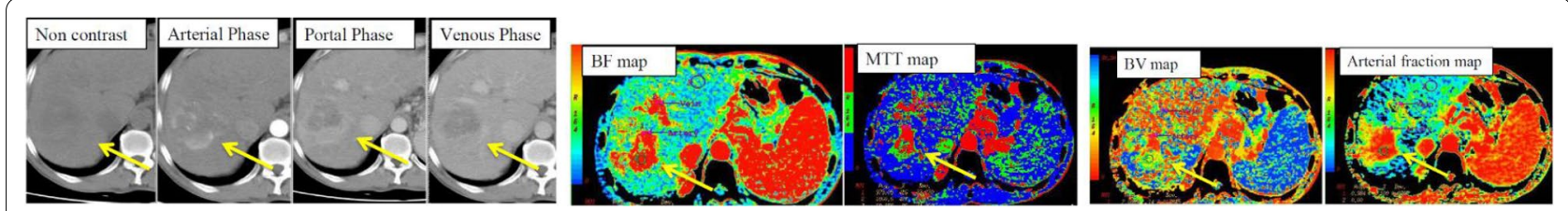

Fig. 3 53-year-old male patient with liver cirrhosis and right hepatic lobe segment VIIVIII HCC underwent radiofrequency ablation on January 2019. Follow-up post-intervention triphasic CT was done on February 2019 and revealed evident intra-lesional pathological enhancement, denoting residual/recurrence activity of this lesion. CTP functional maps showed marked increase in blood flow to the lesion measuring about $233 \mathrm{~mL} / 100 \mathrm{~g} / \mathrm{min}$ compared to $67 \mathrm{~mL} / 100 \mathrm{~g} / \mathrm{min}$ in the rest of hepatic parenchyma. In addition, the mean transit time is reduced to become $6 \mathrm{~s}$ 

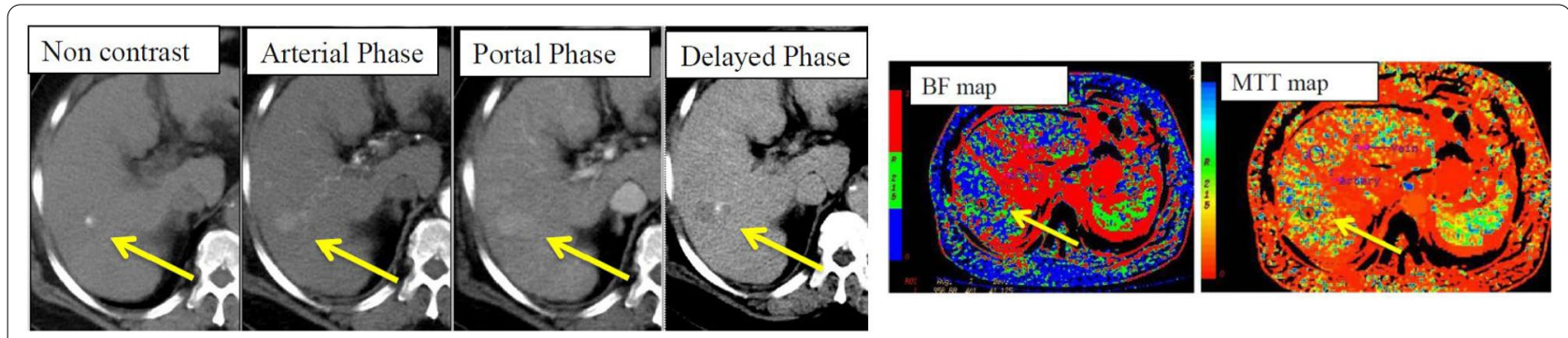

Fig. 4 71-year-old male patient with liver cirrhosis and right hepatic lobe segment VIII HCC underwent TACE on June 2019. Follow-up post-intervention triphasic CT was done on August 2019 and revealed dense injected chemotherapy material "lipidol" showing evidence of pathological enhancement at its lower margin, denoting recurrent/residual activity. CTP functional maps of this recurrent lesional pathological activity showed evident increase in blood flow measuring about $239 \mathrm{~mL} / 100 \mathrm{~g} / \mathrm{min}$ compared to $96 \mathrm{~mL} / 100 \mathrm{~g} / \mathrm{min}$ in the non-ablated hepatic parenchyma. In addition, the mean transit time is reduced to become $8 \mathrm{~s}$

Likewise, at a cut-off level of $\leq 55$, HU arterial had a sensitivity of $100 \%$ and specificity of $100 \%$. In RFA -treated lesions, at a cut-off level of $\leq 53$, $\mathrm{HU}$ porto venous had a sensitivity of $63.64 \%$ and specificity of $80 \%$ (Table 3 ).

\section{Discussion}

Despite the critical importance of early identification of recurrent HCC lesions after loco regional interventions, the currently available imaging modalities are limited by low diagnostic performance and unavailability in some centers. In this study, we investigated the performance of CT perfusion parameters to assess and evaluate HCC response to RFA and TACE. Overall, we found that the $\mathrm{BV}$, transit time and PS yielded high sensitivities and specificities for the assessment of response to TACE.

The outcomes of loco regional treatment of HCC depend primarily on complete ablation of the focal malignant lesions and early identification of residual or local recurrence. Late identification of recurrent lesions can compromise the retreatment outcomes due to the peripheral spread of re-growing lesions [17]. Nonetheless, early identification of recurrent lesions is still challenging, with no standardization across various centers. Particularly, the identification of recurrent HCC lesions with traditional CT following TACE or RFA can be difficult for multiple reasons, including the fact the necrosis of malignant cells may not be associated with a proportional reduction in the tumor size and the possible confusion between RFA-induced reactive hyperemia and recurrent nodules [18]. Besides, in the case of TACE, the identification of $\mathrm{HCC}$ recurrence may be concealed by the beam hardening artifacts around the accumulated lipiodol due to high attenuation from the surrounding vasculature or cellular disruption $[19,20]$. Moreover, the recurrent/residual lesions can lack enhancement on the traditional CT within the first three months after ablation and may be falsely interpreted as successful treatment [21]. Thus, CT perfusion has emerged as a promising modality for assessing patients' response to loco regional interventions; it is defined as "real" blood flow through a volume of tissue per time." The CT perfusion can assess the change in small vessels through serial density measurements [22]. The CT perfusion quantitatively evaluates the hemodynamics changes within the malignant tissue. Thus, it can accurately reflect the pathological changes in the vascular of malignant hepatic tissue [23, 24]. Malignant tissues usually show reduced BF, BV, and PS immediately after treatment [25].

Our study assessed the potential role of CT perfusion technique in the detection of blood flow changes related to the therapeutic effects in $\mathrm{HCC}$ lesions treated with RFA and TACE. According to our results, perfusion parameters, particularly $\mathrm{BF}$ and $\mathrm{BV}$, are higher in the residual disease than in the ablated zone. Such findings can be attributed to the fact that BF is strongly related to the existence of residual or new vessels within the lesions, prompting tumor recurrence; thus, increased BF donates hyper vascularity [15]. On the contrary, transit time was lower in recurrent lesions due to the concurrent reduction in portal venous branches in the residual viable neoplastic tissue. Our results are even consistent with those previously reported in other studies.

In 2015, Chen et al. evaluated changes in perfusion parameters before and after TACE and found that perfusion parameters, particularly HAP and hepatic arterial fraction, increased after treatment in patients with progressive disease.

Our results agree with Mahnken et al. 2013 who reported the usefulness of quantitative volumetric arterial enhancement fraction color mapping for the early detection of recurrent tumor after RFA. They observed that the tumor arterial perfusion values were significantly higher when compared with liver parenchyma. 


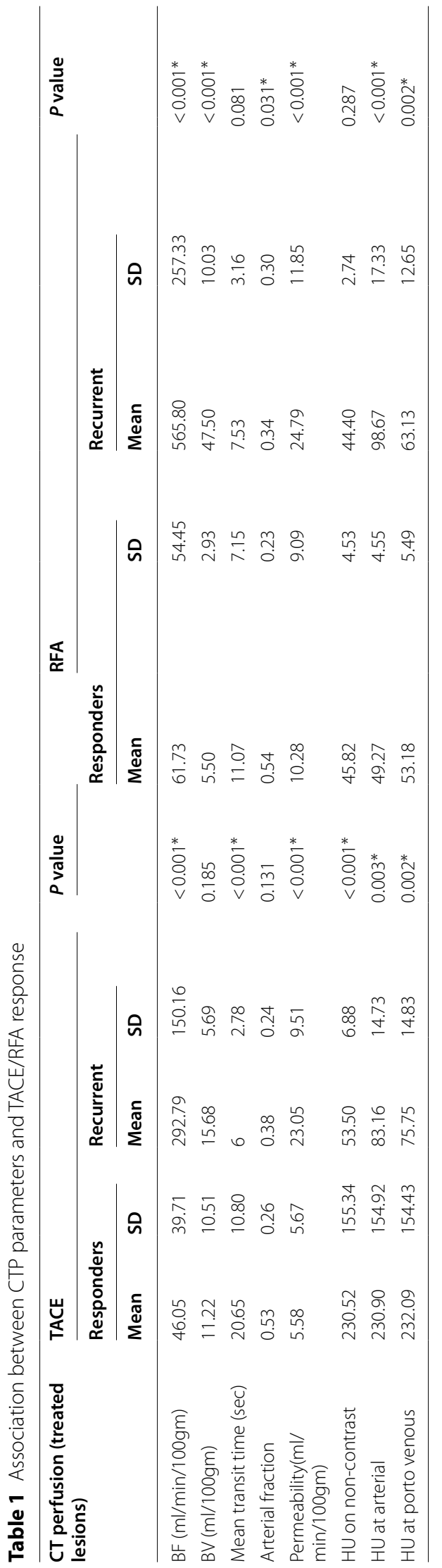


Table 2 Association between CTP parameters and ablated/cirrhotic lesions

\begin{tabular}{|c|c|c|c|c|c|c|c|c|c|c|}
\hline \multirow[t]{3}{*}{ CT perfusion } & \multicolumn{4}{|c|}{ TACE responder } & \multirow[t]{3}{*}{$P$ value } & \multicolumn{4}{|c|}{ RFA Responder } & \multirow[t]{3}{*}{$P$ value } \\
\hline & \multicolumn{2}{|c|}{ Ablated lesions } & \multicolumn{2}{|c|}{$\begin{array}{l}\text { Cirrhotic } \\
\text { parenchyma }\end{array}$} & & \multicolumn{2}{|c|}{ Ablated lesions } & \multicolumn{2}{|c|}{$\begin{array}{l}\text { Cirrhotic } \\
\text { parenchyma }\end{array}$} & \\
\hline & Mean & SD & Mean & SD & & Mean & SD & Mean & SD & \\
\hline $\mathrm{BF}(\mathrm{ml} / \mathrm{min} / 100 \mathrm{gm})$ & 46.05 & 39.71 & 248.84 & 180.82 & $<0.0001^{*}$ & 61.73 & 54.45 & 283.05 & 226.65 & $0.0002^{*}$ \\
\hline BV $(\mathrm{ml} / 100 \mathrm{gm})$ & 11.22 & 10.51 & 16.71 & 9.71 & $0.0213^{*}$ & 5.50 & 2.93 & 25.37 & 12.56 & $<0.0001^{*}$ \\
\hline Mean transit time (sec) & 20.65 & 10.80 & 10.98 & 7.001 & $<0.0001^{*}$ & 11.07 & 7.15 & 10.23 & 4.89 & 0.4270 \\
\hline Arterial fraction & 0.53 & 0.26 & 0.27 & 0.21 & $<0.0001^{*}$ & 0.54 & 0.23 & 0.42 & 0.24 & 0.1420 \\
\hline Permeability(ml/min/100gm) & 5.58 & 5.67 & 23.62 & 16.23 & $<0.0001^{*}$ & 10.28 & 9.09 & 29.44 & 9.77 & $<0.0001^{*}$ \\
\hline HU on non-contrast & 230.52 & 155.34 & 51.05 & 7.20 & $<0.0001^{*}$ & 45.82 & 4.53 & 53.32 & 3.30 & $<0.0001^{*}$ \\
\hline $\mathrm{HU}$ at arterial & 230.90 & 154.92 & 55.62 & 7.22 & $<0.0001^{*}$ & 49.27 & 4.55 & 59.32 & 4.49 & $<0.0001^{*}$ \\
\hline $\mathrm{HU}$ at porto venous & 232.09 & 154.43 & 86.05 & 12.33 & $0.0003^{*}$ & 53.18 & 5.49 & 100.18 & 17.56 & $<0.0001^{*}$ \\
\hline
\end{tabular}

Table 3 Predictive value of CT perfusion for Response in TACE-/RFA-treated lesions by Receiver Operating Characteristic Curve (ROC)

\begin{tabular}{|c|c|c|c|c|c|c|c|c|c|c|}
\hline \multirow[t]{2}{*}{ CT perfusion (treated lesions) } & \multicolumn{5}{|l|}{ TACE } & \multicolumn{5}{|l|}{ RFA } \\
\hline & AUC & Cut off & Sens\% & Spec\% & $P$ value & AUC & Cut off & Sens\% & Spec\% & P-value \\
\hline $\mathrm{BF}(\mathrm{ml} / \mathrm{min} / 100 \mathrm{gm})$ & 0.968 & $\leq 122$ & 100 & 83.3 & $<0.0001^{*}$ & 1 & $\leq 170$ & 100 & 100 & $<0.0001^{*}$ \\
\hline BV (ml/100gm) & 0.685 & $\leq 9$ & 71.43 & 91.67 & 0.0691 & 1 & $\leq 11$ & 100 & 100 & $<0.0001^{*}$ \\
\hline Mean transit time (sec) & 0.933 & $>10$ & 90.48 & 100 & $<0.0001^{*}$ & 0.598 & $>13.9$ & 36.36 & 100 & 0.2977 \\
\hline Arterial fraction & 0.671 & $>0.4$ & 76.19 & 50 & 0.0771 & 0.703 & $>0.2$ & 86.36 & 66.67 & 0.0537 \\
\hline Permeability(ml/min/100gm) & 0.938 & $\leq 14$ & 100 & 83.33 & $<0.0001^{*}$ & 0.806 & $\leq 11$ & 77.27 & 80 & $0.0001^{*}$ \\
\hline HU non contrast & 1 & $>68$ & 100 & 100 & $<0.0001^{*}$ & 0.618 & $>49$ & 36.36 & 100 & 0.2017 \\
\hline HU arterial & 0.980 & $>104$ & 90.48 & 100 & $<0.0001^{*}$ & 1 & $\leq 55$ & 100 & 100 & $<0.0001^{*}$ \\
\hline HU porto venous & 0.998 & $>98$ & 95.24 & 100 & $<0.0001$ & 0.773 & $\leq 53$ & 63.64 & 80 & $0.0005^{*}$ \\
\hline
\end{tabular}

Ippolito et al. 2014 and Choi et al. 2015 reported that CTP parameters obtained 1 week after TACE did not help predict future recurrence. The recurrence eventually occurred at 4 weeks after TACE. The recurrent HCC within TACE-treated areas had significantly higher HAP, and HPI than the lipiodolized area or the cirrhotic parenchyma.

Ren et al. in 2014 showed the usefulness of CT perfusion for early response assessment following anticancer treatment in 100 mice with human colon cancer xenografts. Blood flow, blood volume, and flow extraction product of the tumors were significantly reduced as early as 1 day after treatment with either an antiangiogenic agent (bevacizumab) or a single radiation treatment. Those changes in perfusion parameters occurred significantly faster than changes in the tumor volume. These data suggest that $\mathrm{CT}$ perfusion parameters can be used as biomarkers for earlier post-treatment response assessment before overt changes in tumor size occur.

In patients, Xiong et al. (2010) and Ng et al. (2011) showed a significant decrease in blood flow and/or blood volume after one cycle of the antiangiogenic drug $\mathrm{SU}$ in six patients with liver metastases and as early as 2 days following antiangiogenic treatment with bevacizumab in 24 patients with metastatic carcinoid tumor, respectively.

Following local-regional therapy, Kan et al. in (2005) demonstrated a significant decrease in blood flow, blood volume, and permeability, while showing a significant increase in MTT immediately and 2 days after TACE in 14 rats with liver metastasis (from murine mammary cancer cells).

In patients with HCC, Yang et al. in 2011 demonstrated a significant decrease in HAP and HPI in HCCs at 4 weeks after TACE.

These studies show a potential role of perfusion CT for more accurate assessment of treatment response compared with traditional morphologic imaging,

Our study does not agree with Spira et al. 2012 who reported that the liver is probably the most challenging organ for CTP measurement and interpretation due to considerable diaphragmatic movement during breathing, as the motion correction and the application of filters allow reconstruction of good-quality images even with free-breathing of the patient during scanning. 
We acknowledge that the present study has some limitations. We used the 16-slice CT scanner, which allows for $2 \mathrm{~cm}$ coverage only for each patients; thus, it covers the treated lesion only. Besides, we did not compare the CT parameters with histological evaluation of vessel density, the standard reference for evaluation of response to TACE and RFA. Our study is also limited by the relatively small sample size and being a singlecenter experience. Thus, our generalizability is limited and our results have to be confirmed by further analysis in greater cohorts of patients.

\section{Conclusions}

The present study confirms the feasibility of CT perfusion for assessment of response to TACE and RFA among patients with HCC. We found that the CT perfusion parameters, particularly BF, yielded high diagnostic performance for prediction of response and early recurrence. CT perfusion showed a significant role in distinguishing between responding and recurrent lesions. Nonetheless, our results have to be confirmed by further analysis in greater cohorts of patients.

\begin{abstract}
Abbreviations
AASLD: American Association for the Study of Liver Diseases; ACR: American College of Radiology; AFP: Alpha-fetoprotein; BF: Blood flow; BV: Blood volume; CECT: Contrast-enhanced computed tomography; CR: Complete response; CT: Computed tomography; CTP: Computed tomography perfusion; CTACE: Conventional Transarterial chemoembolization; DSA: Digital Subtraction Angiography; FN: False negative fraction; FP: False positive fraction; HAP: Hepatic arterial phase; HBV: Hepatitis B virus; HCC: Hepatocellular carcinoma; HCV: Hepatitis C virus; HFL: Hepatic focal lesion; INR: International normalized ratio; LIRADS: Liver imaging reporting and data system; MDCT: Multi-detector computed tomography; MTT: Mean transit time; MWA: Microwave ablation; mRECIST: Modified RECIST; PD: Progressive disease; PR: Partial response; PS: Permeability surface; PPV: Positive predictive value; PVE: Portal vein embolization; RCT: Randomized control trial; RECIST: Response Evaluation Criteria in Solid Tumors; RFA: Radiofrequency ablation; RN: Regenerative nodule; ROI: Region of interest; SD: Stable disease; SD: Standard deviation; SIR: Society of Interventional Radiology.
\end{abstract}

\section{Acknowledgements}

Not applicable.

\section{Authors' contributions}

MFO put the idea of the study, did CTP assessment, and performed the statistical analysis. IHS participated in the study design and CTP assessment. AH and ME involved in data collection and clinical assessment of the patients. LIA edited the manuscript, participated in the study design, and performed the CTP assessment. All authors read and approved the final manuscript.

\section{Funding}

Not applicable (no funding).

\section{Availability of data and material}

All the datasets used and analyzed in this study are available with the corresponding author on reasonable request.

\section{Declarations}

Ethics approval and consent to participate

All patients were submitted to informed consent including examination description and benefits. The present manuscript was approved by the local ethics committee of Cairo University Hospital [D-23-2019] and prepared in concordance with the recommendations of STrengthening the Reporting of OBservational studies in Epidemiology (STROBE) (16).

\section{Consent for publication}

All adult patients included in this research gave written informed consent to publish the data contained within this study.

\section{Competing interests}

The authors declare that they have no financial or nonfinancial competing interest.

\section{Author details}

${ }^{1}$ Diagnostic and Interventional Radiology, Cairo University, Cairo, Egypt. ${ }^{2}$ Internal Medicine, Cairo University, Cairo, Egypt.

Received: 13 Auqust 2021 Accepted: 11 November 2021

Published online: 23 November 2021

\section{References}

1. Anugwom CM, Allaire M, Akbar SMF, Sultan A, Bollipo S, Mattos AZ, Debes JD (2021) Hepatitis B-related hepatocellular carcinoma: surveillance strategy directed by immune-epidemiology. Hepatoma Res 7

2. Llovet JM, Kelley RK, Villanueva A, Singal AG, Pikarsky E, Roayaie S, Lencioni R, Finn RS (2021) Hepatocellular carcinoma. Nat Rev Dis Primers 7:6

3. Abudeif A (2019) Epidemiology and risk factors of Hepatocellular Carcinoma in Egypt. Sohag Med J 23(3):8-12

4. Rashed WM, Kandeil MAM, Mahmoud MO, Ezzat S (2020) Hepatocellular Carcinoma (HCC) in Egypt: a comprehensive overview. J Egypt Natl Canc Inst 32(1):1-11

5. Akinyemiju T, Abera S, Ahmed M, Alam N, Alemayohu MA, Allen C, Burdenof Disease Liver Cancer Collaboration G (2017) The burden of primary liver cancer and underlying etiologies from 1990 to 2015 at the global, regional, and national level: results from the global burden of disease study 2015. JAMA Oncol 3(12):1683-1691

6. Schulze K, Imbeaud S, Letouzé E, Alexandrov LB, Calderaro J, Rebouissou S, Zucman-Rossi J, et al (2015) Exome sequencing of hepatocellular carcinomas identifies new mutational signatures and potential therapeutic targets. Nat Genet 47(5):505-511

7. Bayard Q, Meunier L, Peneau C, Renault V, Shinde J, Nault JC, Letouzé E et al (2018) Cyclin A2/E1 activation defines a hepatocellular carcinoma subclass with a rearrangement signature of replication stress. Nat Commun 9(1):1-14

8. Sanyal AJ, Yoon SK, Lencioni R (2010) The etiology of hepatocellular carcinoma and consequences for treatment. Oncologist 15:14-22

9. Pomej K, Scheiner B, Park D, Bauer D, Balcar L, Meischl T, Pinter M (2020) Vascular complications in patients with hepatocellular carcinoma treated with sorafenib. Cancers 12(10):2961

10. European Association For The Study Of The Liver (2018) EASL clinical practice guidelines: management of hepatocellular carcinoma. J Hepatol 69(1):182-236

11. Ko KL, Mak LY, Cheung KS, Yuen MF (2020) Hepatocellular carcinoma: recent advances and emerging medical therapies. F1000Research, 9

12. Lencioni R, Llovet JM (2010) Modified RECIST (mRECIST) assessment for hepatocellular carcinoma. In: Seminars in liver disease (vol 30, no. 01, pp 052-060). (C) Thieme Medical Publishers

13. Ippolito D, Capraro C, Casiraghi A, Cestari C, Sironi S (2012) Quantitative assessment of tumour associated neovascularisation in patients with liver cirrhosis and hepatocellular carcinoma: role of dynamic-CT perfusion imaging. Eur Radiol 22(4):803-811 
14. Chen ML, Zeng QY, Huo JW, Yin XM, Li BP, Liu JX (2009) Assessment of the hepatic microvascular changes in liver cirrhosis by perfusion computed tomography. World J Gastroenterol: WJG 15(28):3532

15. Shalaby MH, Shehata KAA (2017) CT perfusion in hepatocellular carcinoma: Is it reliable? Egypt J Radiol Nuclear Med 48(4):791-798

16. Von Elm E, Altman DG, Egger M, Pocock SJ, Gøtzsche PC, Vandenbroucke JP, Initiative S (2014) The strengthening the reporting of observational studies in epidemiology (STROBE) Statement: guidelines for reporting observational studies. Int J Surg 12(12):1495-1499

17. Ippolito D, Fior D, Bonaffini PA, Capraro C, Leni D, Corso R, Sironi S (2014) Quantitative evaluation of CT-perfusion map as indicator of tumor response to transarterial chemoembolization and radiofrequency ablation in HCC patients. Eur J Radiol 83(9):1665-1671

18. Kim S, Mannelli L, Hajdu CH, Babb JS, Clark TW, Hecht EM, Taouli B (2010) Hepatocellular carcinoma: assessment of response to transarterial chemoembolization with image subtraction. J Magn Resonance Imaging 31(2):348-355

19. Mannelli L, Kim S, Hajdu CH, Babb JS, ClarkTW, Taouli B (2009) Assessment of tumor necrosis of hepatocellular carcinoma after chemoembolization: diffusion-weighted and contrast-enhanced MRI with histopathologic correlation of the explanted liver. Am J Roentgenol 193(4):1044-1052

20. Kamel IR, Bluemke DA, Eng J, Liapi E, Messersmith W, Reyes DK, Geschwind JFH (2006) The role of functional MR imaging in the assessment of tumor response after chemoembolization in patients with hepatocellular carcinoma. J Vasc Interv Radiol 17(3):505-512
21. Ippolito D, Bonaffini PA, Capraro C, Leni D, Corso R, Sironi S (2013) Viable residual tumor tissue after radiofrequency ablation treatment in hepatocellular carcinoma: evaluation with CT perfusion. Abdom Imaging 38(3):502-510

22. Morsbach F, Pfammatter T, Reiner CS, Fischer MA, Sah BR, Winklhofer S, Alkadhi $\mathrm{H}$ et al (2013) Computed tomographic perfusion imaging for the prediction of response and survival to transarterial radioembolization of liver metastases. Invest Radiol 48(11):787-794

23. Chen G, Ma DQ, He W, Zhang BF, Zhao LQ (2008) Computed tomography perfusion in evaluating the therapeutic effect of transarterial chemoembolization for hepatocellular carcinoma. World J Gastroenterol:WJG 14(37):5738

24. Popovic APP, Leban A, Kregar K, Garbajs M, Dezman R, Bunc M (2018) Computed tomographic perfusion imaging for the prediction of response and survival to transarterial chemoembolization of hepatocellular carcinoma. Radiol Oncol 52(1):14

25. Haj-Mirzaian A, Kadivar A, Kamel IR, Zaheer A (2020) Updates on imaging of liver tumors. Curr Oncol Rep 22(5):1-10

\section{Publisher's Note}

Springer Nature remains neutral with regard to jurisdictional claims in published maps and institutional affiliations.

\section{Submit your manuscript to a SpringerOpen ${ }^{\circ}$ journal and benefit from:}

- Convenient online submission

- Rigorous peer review

- Open access: articles freely available online

- High visibility within the field

- Retaining the copyright to your article

Submit your next manuscript at $\boldsymbol{\nabla}$ springeropen.com 